\title{
A Novel Travelling Time Estimation Mechanism for Minimizing Unnecessary Handovers from Cellular Networks to WLANs
}

\author{
Mahmood, A., Zen, H., Othman, A. K. \\ Faculty of Engineering, Universiti Malaysia Sarawak \\ Kota Samarahan, 94300, Sarawak, Malaysia
}

\author{
Siddiqui, S. A. \\ University of Jinan \\ Jinan, Shandong, 250022, People's Republic of China
}

\begin{abstract}
The 'Beyond 3G Wireless Networks Architecture' proposes to assimilate numerous heterogeneous wireless network technologies over an Internet Protocol Backbone. However, in order to offer seamless mobility, cost - effective connectivity and desired Quality of Service (QoS) in such a diverse environment; efficacious vertical handover support still remains a major design challenge. In this research/manuscript, a novel handover decision mechanism, 'Travelling Time Prediction Based on the Consecutive RSS Measurements' in an IEEE 802.11 WLAN cell is suggested. The method uses a time threshold which is computed by a Mobile Terminal (MT) as soon as it penetrates into a WLAN boundary. The estimated travelling time is then compared with the time threshold, so as to make handover decisions for minimizing the probability of unnecessary handovers. Our performance analysis reveals that the suggested mechanism effectively reduces number of unnecessary handovers by $60 \%$ as compared to the already proposed schemes.
\end{abstract}

Keywords-Vertical Handover Decisions; Received Signal Strength; Quality of Service; Handover Failures; Unnecessary Handovers

\section{INTRODUCTION}

The increased proliferation of numerous wireless network technologies [i.e. 2G, 3G, IEEE802.11 WLAN, IEEE802.16 WiMAX, etc.) and the unfolding of multi - interface mobile devices has created a user demand to seamlessly roam anywhere at anytime. One considerable advancement obsessed by this ubiquitous wireless access is evolvement of $4 \mathrm{G}$ Systems, which represents heterogeneous wireless environment possessing disparate / diverse access network technologies with a varying bandwidth, latency and cost. In such sort of a networking scenario, MTs with multiple interfaces opt for the befitting access links among the most accessible substitutes (in addition to the conventional cellular telephony networks), with no perceived disruption to an ongoing video and voice conversation [1 - 3]. Thus, this capability to seamlessly handover amongst the heterogeneous wireless networks is regarded as the 'vertical handover'. It is differentiated from the 'horizontal handover', which occurs as MTs traverses between point of attachments (PoA) supporting the same network technology [4-7]. The IEEE802.21 standard proposes a framework so as to address challenges for facilitating seamless mobility in heterogeneous access networks; however, real algorithms to be employed are left over for the consideration of designers [8 - 9].

'Vertical Handover Processes' can be segmented into three phases - Network Detection, Handover Decision and the Handover Triggering [9 - 10]. Numerous factors have been deliberated in the literature for use in the Vertical Handover Decision algorithms. These included received signal strength (RSS), available bandwidth, power consumption, network connection time, handover latency, security, MTs mobility, monetary costs, user preferences, etc. [11 - 13].

In this manuscript, a Vertical Handover Decision scheme has been proposed for reducing probability of unnecessary handovers from $3 \mathrm{G}$ cellular networks to the WLANs, so as to provide optimal network utilization and an enhanced user satisfaction. Performance analysis depict(s) that the proposed scheme yields successful results as compared to techniques already proposed in the literature.

The paper is organized as follows: Section II provides an outline of similar studies already proposed in the literature; Section III presents an algorithm for anticipating travelling time in a WLAN cell based on successive RSS measurements, and a time threshold estimation algorithm for minimizing the unnecessary handovers; whereas, the Section IV depicts the performance(s) of proposed mechanism.

\section{LITERATURE REVIEW}

Existing literature reports a number of associated studies pertinent to the Vertical Handover Decisions. In [4], a cross layer design for providing context awareness and a mobility control is presented for heterogeneous wireless IP networks. Similarly, a mechanism for computing dynamic boundary area based on the velocity of MT and WLAN cell dimension is presented in [5]; wherein, handover(s) from the WLAN to $3 \mathrm{G}$ cellular system is initiated as soon as the MT penetrates the WLAN boundary and the handover process is accomplished before MT exits WLAN area. This is also true for the mobility architectures suggested in [6] and [7]. In [8], a handover necessity estimation scheme is proposed for minimizing the unnecessary handovers based on the dwell time estimation and computation of the threshold values. The said mechanisms are quite effective for handoffs commencing from WLAN to $3 \mathrm{G}$ networks; nonetheless, it is not considered efficacious for 